\title{
Vacuum-polarization contribution to the hyperfine-structure splitting of hydrogenlike atoms
}

\author{
S. M. Schneider and W. Greiner \\ Institut für Theoretische Physik, Johann Wolfgang Goethe Universität, Postfach 111 932, \\ D-60054 Frankfurt am Main, Germany
}

\author{
G. Soff \\ Gesellschaft für Schwerionenforschung, Planckstraße 1, Postfach 110 552, D-64220 Darmstadt, Germany \\ and Institut für Theoretische Physik, Technische Universität Dresden, Mommsenstraße 13, D-01062 Dresden, Germany
}

(Received 13 December 1993)

\begin{abstract}
A calculation of the vacuum-polarization contribution to the hyperfine splitting for hydrogenlike atoms is presented. The extended nuclear charge distribution is taken into account. For the experimentally interesting case ${ }^{209} \mathrm{Bi}^{82+}$ we predict a $\Delta \lambda=-1.6 \mathrm{~nm}$ shift for the transition wavelength of the ground-state hyperfine splitting.

PACS number(s): 31.30. Gs
\end{abstract}

\section{INTRODUCTION}

The ground-state hyperfine splitting of hydrogen, the well-known $21-\mathrm{cm}$ radiation, has been measured with a relative accuracy up to $10^{-12}$ [1]. However, the accuracy of the theoretically predicted value of the transition wavelength is about six orders of magnitude lower [1]. There are various corrections to the first-order perturbation theory calculation of the hyperfine splitting: radiative corrections, recoil and radiative recoil corrections, and nuclear structure corrections. The latter can be divided into polarizability corrections and modifications due to the extended nuclear charge-current distribution which has been computed by Zemach [2] in the nonrecoil limit, i.e., $m_{N} \rightarrow \infty$. For heavy nuclei the finite-nuclearsize contribution will dominate, whereas recoil corrections are assumed to be relatively negligible. QED corrections become more and more important for heavy elements because of the large effective coupling constant $Z \alpha$ to the electrostatic field of the nucleus.

Experimental as well as theoretical data on the hyperfine structure of hydrogenlike high- $Z$ atoms were not available up to now. Precision calculations of this level splitting have been motivated by recent experiments with hydrogenlike high-Z atoms, e.g., in Ref. [3], where ${ }^{209} \mathrm{Bi}^{82+}$ is under examination [3]. From the first successful experiment of Klaft et al. one deduced a ground-state transition wavelength of $\Delta \lambda_{\text {expt }}=243.87(4) \mathrm{nm}$ [4]. For the next experimental generation one is aiming at an accuracy of about $10^{-6}$ [5], which represents a severe challenge for the theoretical description.

Recently, two groups presented a first-order perturbation theory calculation of the hyperfine structure of hydrogenlike ${ }^{209} \mathrm{Bi}[6,7]$. The authors concentrated on the effects of the extended nuclear charge-current distribution. The modification according to the finite nuclear charge distribution is known as the Breit-Schawlow effect while the correction due to the extended magnetization distribution is the Bohr-Weisskopf effect [8].

Our paper represents the first part of a detailed analysis of the first-order radiative corrections to the hyperfine structure of hydrogenlike atoms. Here, we focus our attention on the vacuum-polarization contribution. An accurate evaluation of the self-energy level shift is in progress.

\section{THE HYPERFINE-STRUCTURE SPLITTING}

First we summarize briefly the results of the first-order calculation for the most interesting case ${ }^{209} \mathrm{Bi}^{82+}$. In first-order perturbation theory the hyperfine-structure splitting follows from [9]

$$
\begin{aligned}
\Delta E= & \frac{2 \kappa}{4 k^{2}-1} e g_{N} \mu_{N}[F(F+1)-I(I+1)-j(j+1)] \\
& \times\left.\left(2 \int_{0}^{\infty} G(r) F(r) \frac{1}{r^{2}} d r\right)\right|_{\left(F_{1}\right)-\left(F_{2}\right)}
\end{aligned}
$$

where $I$ and $j$ are the total spin of the nucleus and the electron, respectively, and $F$ designates the total spin of the electron-nucleus system. $g_{N}$ is the anomalous magnetic moment and $\mu_{N}$ denotes the nuclear magneton. For ${ }^{209} \mathrm{Bi}^{82+}$, the angular momentum quantum numbers are $I=\frac{9}{2}$ and $j=\frac{1}{2}$, resulting in the two values $F_{1}=5$ and $F_{2}=4$. $\kappa$ signifies the Dirac angular-momentum quantum number and $k=l$ for $\kappa>0$ or $k=l+1$ for $\kappa<0$, where $l$ is the orbital angular-momentum quantum number of the electron. The magnetic moment of bismuth is taken to be $\mu_{\mathrm{Bi}}=4.1106 \mu_{N}$ [10]. Furthermore, $G(r)$ and $F(r)$ are the relativistic radial wave functions, satisfying the radial Dirac equations

$$
\begin{aligned}
& \frac{d G(r)}{d r}=-\frac{\kappa}{r} G(r)+\left[E+m_{e}-V(r)\right] F(r), \\
& \frac{d F(r)}{d r}=\frac{\kappa}{r} F(r)-\left[E-m_{e}-V(r)\right] G(r) .
\end{aligned}
$$

The electrostatic potential $V(r)$ is generated by the spherical symmetric nuclear charge distribution.

For point-nucleus wave functions the integral in Eq. (1) is known analytically, e.g., it reads for the $1 s_{1 / 2}$ state [9] 


$$
\begin{aligned}
\int_{0}^{\infty} G^{\text {p.n. }}(r) F^{\text {p.n. }} & (r) \frac{1}{r^{2}} d r \\
= & \frac{(Z \alpha)^{3}}{\sqrt{1-(Z \alpha)^{2}}\left[2 \sqrt{1-(Z \alpha)^{2}}-1\right]} .
\end{aligned}
$$

For extended nuclear charge distributions this integral has to be determined numerically. For our explicit calculations of the general behavior we utilized wave functions corresponding to the Coulomb field of a homogeneously charged sphere. The root-mean-square radii of the different nuclei are tabulated in Ref. [11]. For nuclei with $Z>30$ we found that the difference between results for a point nucleus and an extended nucleus may be parametrized as

$$
\Delta E(\text { finite })=\left(1-e^{A+B \cdot Z}\right) \Delta E(\text { point }),
$$

where $A=-6.813$ and $B=5.5884 \times 10^{-2}$. For bismuth this implies a $12 \%$ modification. In lowest order the transition wavelength between the $F_{1}=I+\frac{1}{2}$ and the $F_{2}=I-\frac{1}{2}$ states of ${ }^{209} \mathrm{Bi}^{82+}$ was computed to be $\Delta \lambda^{1}=238.8 \mathrm{~nm}$, employing an experimentally determined Fermi distribution [12]. An examination of the BohrWeisskopf effect yielded a shift of $\Delta \lambda^{\mathrm{BW}} \simeq+3.5 \mathrm{~nm}$ [7]. This value was determined within the extreme singleparticle model and might be still uncertain. For muonic atoms Johnson and Sorensen [13] presented an evaluation within the configuration mixing model that coincides with the experimental value from Rüetschli et al. [14]. Within the framework of the single-particle model the Bohr-Weisskopf effect is assumed to be underestimated because of missing configuration mixing contributions. Thus, a more sophisticated calculation of the BohrWeisskopf effect for the hydrogenlike Bi nucleus seems to be vital for a more rigorous theoretical prediction.

\section{RADIATIVE CORRECTIONS}

The problem of an electron moving in the central field of a nucleus belongs to the more general bound-state QED. The QED corrections to the hyperfine splitting generally are calculated in the nonrecoil limit $\left(m_{e} / m_{N} \rightarrow 0\right)$, where the nucleus is reduced to an external electromagnetic field. For hydrogen or very light elements the QED corrections to the bound electron, e.g., the Lamb shift, traditionally are expanded in the effective coupling constant $Z \alpha$. In the context of the hyperfine structure pioneering investigations were performed by Kroll and Pollock [15] and by Karplus and Klein [16], who calculated the level splitting in a series expansion to order $\alpha(Z \alpha)$. This series expansion was later extended by Brodsky and Ericson [17] and by Sapirstein [18]. Unfortunately, these extensive calculations are not applicable to medium- $Z$ or high- $Z$ atoms, since the series expansion would converge rather slowly when $Z \alpha$ approaches unity. This behavior was also discovered in the computation of the Lamb shift. For that reason, methods were developed to treat the propagator in the spherical symmetric Coulomb field of the nucleus exact to all orders in $Z \alpha$.

To compute radiative corrections for bound electrons within the external field approximation one is led to the
Furry or bound-state interaction picture, where the external field is included in the electron field operator as well as in the Green function of the Dirac equation. The graphical representation of the first-order radiative corrections within the Furry picture is shown in Figs. $1(a)-1(c)$. The double lines signify wave functions and propagators, respectively, that are exact in the electromagnetic field of the nucleus. A direct application of the methods developed for the evaluation of the Lamb shift is not possible because of the nonspherical symmetry of the external field. Since the magnetic field of the nucleus is rather small compared with the electrostatic potential, it is legitimate to treat it as a perturbation. Thus, the vacuum-polarization part of the QED corrections [Fig. 1(c)] can be expanded in terms of the magnetic coupling. In Fig. 2 the diagrammatic depiction of this expansion is displayed. Two contributions arise if one restricts the calculation to a single magnetic coupling: (a) the diagram with one loop that couples to the magnetic field, which appears due to the expansion of the exact propagator, and (b) two diagrams with one magnetic coupling from the expansion of the exact wave function. In Fig. 2 the thick lines symbolize the exact propagator or wave function in the Coulomb-like electrostatic field. In our approximate numerical elaborations the loop propagator is taken in first order in the external field. This transforms diagram $2 a$ to an Uehling-like contribution, which couples to the magnetic field. In Fig. 2(b) the loop part of the diagram reduces to the ordinary Uehling part. We denote these two energy shifts $\Delta E_{\mathrm{ML}}$ and $\Delta E_{\mathrm{EL}}$, where the suffices $M L$ and EL indicate the magnetic loop and the electrostatic loop contribution, respectively. The total energy shift is $\Delta E^{\mathrm{VP}}=\Delta E_{\mathrm{ML}}+\Delta E_{\mathrm{EL}}$.

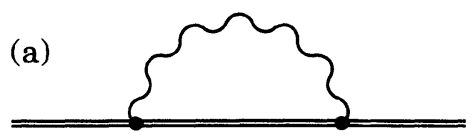

(b)

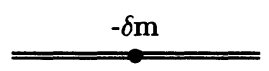

(c)

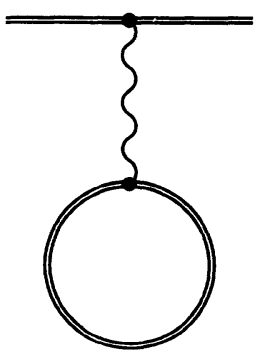

FIG. 1. Graphical representation of radiative corrections to the hyperfine structure within the Furry picture. Double lines indicate exact wave functions and exact propagators in the external electromagnetic field, respectively. (a) is the selfenergy, (b) is the divergent mass-renormalization diagram, which cancels with an identical divergency appearing in (a), and (c) represents the vacuum-polarization part. 
At first we consider the magnetic contribution. In momentum space the induced vacuum-polarization potential $A_{\mu}^{\mathrm{VP}}(q)$ of order $\alpha$ is given by [17]

$$
A_{\mu}^{\mathrm{VP}}(q)=\frac{\alpha}{2 \pi} q^{2} \int_{0}^{1} d v \frac{2 v^{2}\left(1-v^{2} / 3\right)}{4 m_{e}^{2}-q^{2}\left(1-v^{2}\right)} A_{\mu}(q)
$$

where $\mathbf{A}(\mathbf{q})=-2 \delta\left(q_{0}\right) i(\mathbf{q} \times \mathbf{m}) / \mathbf{q}^{2}$. Introducing the Fourier transform $A_{\mu}^{\mathrm{VP}}(x)$ by

$$
A_{\mu}^{\mathrm{VP}}(x)=\int \frac{d^{4} q}{(2 \pi)^{2}} e^{i q \cdot x} A_{\mu}^{\mathrm{VP}}(q),
$$

one obtains the magnetic part of the vacuum-polarization potential

$$
\begin{aligned}
\mathbf{A}^{\mathrm{VP}}(\mathbf{r})= & -\frac{i \alpha}{\pi} \int_{0}^{1} d v \int \frac{d^{3} q}{(2 \pi)^{2}} \\
& \times e^{-i \mathbf{q} \cdot \mathbf{r}}(\mathbf{q} \times \mathbf{m}) \frac{2 v^{2}\left(1-v^{2} / 3\right)}{4 m_{e}^{2}+\mathbf{q}^{2}\left(1-v^{2}\right)}
\end{aligned}
$$

Writing

$$
\mathbf{q} \times \mathbf{m}=\left.i\left(\frac{\partial}{\partial \mathbf{s}} e^{-i \mathbf{q} \cdot \mathbf{s}}\right)\right|_{\mathbf{s}=0} \times \mathbf{m}
$$

and interchanging differentiation and integration, one can make use of the integral formula

$$
\int \frac{d^{3} q}{(2 \pi)^{2}} \frac{e^{i \mathbf{q} \cdot \mathbf{r}}}{a^{2}+\mathbf{q}^{2}}=\frac{1}{2} \frac{e^{-a|\mathbf{r}|}}{|\mathbf{r}|} .
$$

The final expression for the vacuum-polarization vector potential in position space reads

$$
\begin{aligned}
\mathbf{A}^{\mathrm{VP}}(\mathbf{r})=\frac{2 \alpha}{3 \pi} \mathbf{A}(\mathbf{r}) \int_{1}^{\infty} d z & \left(1-\frac{1}{2 z^{2}}\right)^{1 / 2}\left(1+\frac{1}{2 z^{2}}\right) \frac{1}{z} \\
& \times e^{-2 m_{e} r z}\left[2 m_{e} r z+1\right]
\end{aligned}
$$

Here, the vector field $\mathbf{A}(\mathbf{r})=(\mathbf{m} \times \mathbf{r}) / r^{3}$ is the only operator acting on the wave functions. Hence, according to the result within the framework of first-order perturbation theory we deduce that the integral in Eq. (1) has to be exchanged with

$$
\int_{0}^{\infty} G(r) F(r) \frac{1}{r^{2}} d r \rightarrow \frac{2}{3} \frac{\alpha}{\pi} \int_{1}^{\infty} d z\left(1-\frac{1}{2 z^{2}}\right)^{1 / 2}\left(1+\frac{1}{2 z^{2}}\right) \frac{1}{z} \int_{0}^{\infty} d r G(r) F(r) \frac{1}{r^{2}} e^{-2 m_{e} r z}\left[2 m_{e} r z+1\right]
$$

in order to derive the corresponding expression for the vacuum-polarization energy shift. If one expands the analytically known product $G^{\text {p.n. }}(r) F^{\text {p.n. }}(r)$ for the $1 s$ wave function into a series in $Z \alpha$ one obtains $\left(m_{e}=1\right)$

$$
G(r) F(r) \frac{1}{r^{2}}=-2(Z \alpha)^{3} Z \alpha e^{-2 Z \alpha r}\left\{1-\left[\frac{9}{16}+\frac{1}{2}\left(2 \gamma_{E}-3\right)+\ln (2 Z \alpha r)\right](Z \alpha)^{2}+\frac{1}{4}(Z \alpha)^{3} r+\cdots\right\},
$$

where all terms are expanded except for the exponential $e^{-2 Z \alpha r}$. $\gamma_{E}=0.577215 \ldots$ is Eulers constant. If one writes

$$
G(r) F(r) r^{2}=A_{1}+A_{2}(Z \alpha)^{2}+\cdots,
$$

then it is obvious that

$$
A_{1}=-2(Z \alpha)^{3}(Z \alpha) e^{-2 Z \alpha r}=\pi \frac{d}{d r}\left(\Psi^{S}(r)\right)^{2},
$$

where

$$
\Psi^{S}(r)=\left(\frac{(Z \alpha)^{3}}{\pi}\right)^{1 / 2} e^{-Z \alpha r}
$$

is the $1 s$ Schrödinger wave function. So, in the $(Z \alpha)$ expansion of the product of the radial Dirac wave functions, the first term will yield the nonrelativistic formula derived by Zwanziger [19]. The second term represents a first relativistic correction factor. In Figs. 3(a) and 3(b) the magnetic part of the vacuum-polarization energy shift $\Delta E_{\mathrm{ML}}$, normalized to $\Delta E^{1}(3 / 8) \alpha(Z \alpha)$, is presented for different ranges of the nuclear charge number $Z$. The full line signifies the relativistic calculation and the dashed line is addressed to the nonrelativistic calculation with Schrödinger wave functions. Additionally, the dotted line corresponds to the series expansion up to the order $\alpha(Z \alpha)^{2}$ while the dashed-dotted line indicates the computed value incorporating the relativistic correction factor deduced from the Dirac wave function expansion. (a)

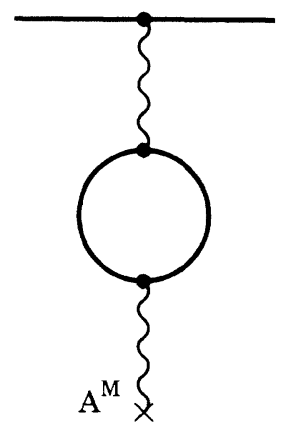

(b)
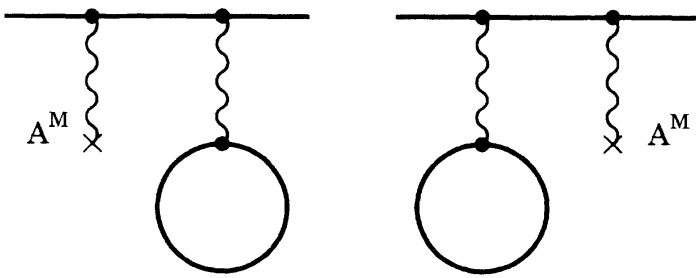

FIG. 2. Depiction of the two resulting contributions from the expansion of the vacuum-polarization correction [Fig. 1(c)] in first order in the external magnetic field. Thick lines refer to exact wave functions or exact propagators in the electrostatic field. The magnetic part (a) results from the expansion of the propagator and (b) is obtained from an expansion of the wave functions. 
One easily verifies that all evaluations yield nearly the same result for hydrogen and that the relativistic correction is not negligible for high- $Z$ nuclei. In contrast to the result given in the paper by Brodsky and Ericson [17] we found that the series expansion of the nonrelativistic point nucleus formula for the magnetic energy shift yields

$$
\Delta E_{\mathrm{ML}}=\Delta E^{F}\left[\frac{3}{8} \alpha(Z \alpha)-\frac{4}{5} \frac{\alpha}{\pi}(Z \alpha)^{2}\right] \text {, }
$$

where $\Delta E^{F}$ denotes the nonrelativistic first-order result of Fermi [20]. The difference to the expression from Brodsky and Ericson is provided by the factor $-\frac{4}{5}$ in front of the $\alpha(Z \alpha)^{2}$ term, which was quoted to be $-\frac{2}{3}$.

As one learned from Uehling-shift calculations, the finite size of the nucleus modifies the point-nucleus results by a significant amount for medium to high- $Z$ atoms. Figure 4 presents a comparison between the relativistic point-nucleus calculation (dashed line) and the relativistic values for extended nuclear charge distributions (full line).
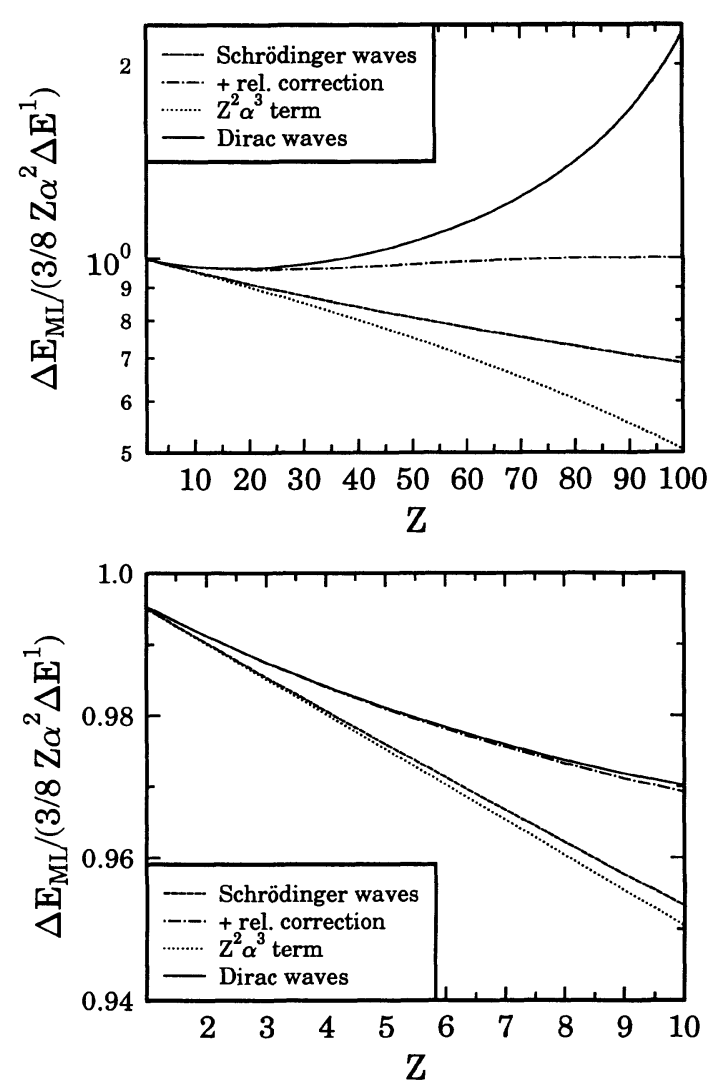

FIG. 3. The magnetic part of the hyperfine splitting energy $\Delta E_{\mathrm{ML}}$, normalized to $\frac{3}{8} \alpha(Z \alpha) \Delta E^{1}$, as a function of the nuclear charge number $Z$. In Fig. 3(a) the full range $1 \leq Z \leq 100$ is considered, Fig. 3(b) shows a magnification of the low- $Z$ area $1 \leq Z \leq 10$. The full line represents the relativistic calculation while the dashed line corresponds to the Schrödinger wavefunction calculation. Additionally, we plot the result for the series expansion up to order $\alpha(Z \alpha)^{2}$ (dotted line) and the relativistically corrected result (dashed-dotted line). All energy shifts are derived for point nuclei.

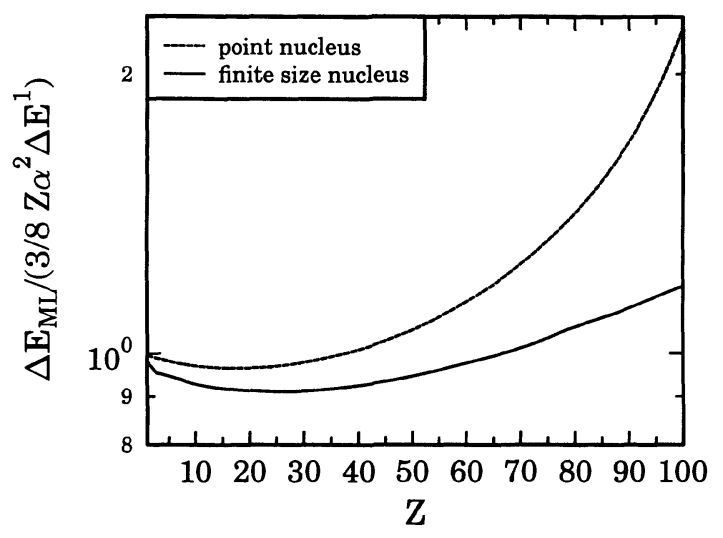

FIG. 4. The splitting energy $\Delta E_{\mathrm{ML}}$ displayed with the same normalization as in Fig. 3. The dashed line refers to the relativistic point nucleus result and should be compared with the full line, which indicates the corresponding value for the extended nucleus. The full line exhibits some slight structures because of the nonanalytical behavior of the nuclear radii. The difference between the result for a point nucleus and an extended nuclear charge distribution is notable and seems to be even more pronounced compared with the outcome of the lowest-order calculation.

The influence of the electrostatic vacuum polarization on the hyperfine splitting is numerically included by adding the Uehling potential to the static potential $V(r)$ entering the Dirac equation. The resulting wave functions are applied to the first-order calculation. The difference to the ordinary first-order result yields $\Delta E_{\mathrm{EL}}$.

In Fig. 5 the total normalized energy shift, summing

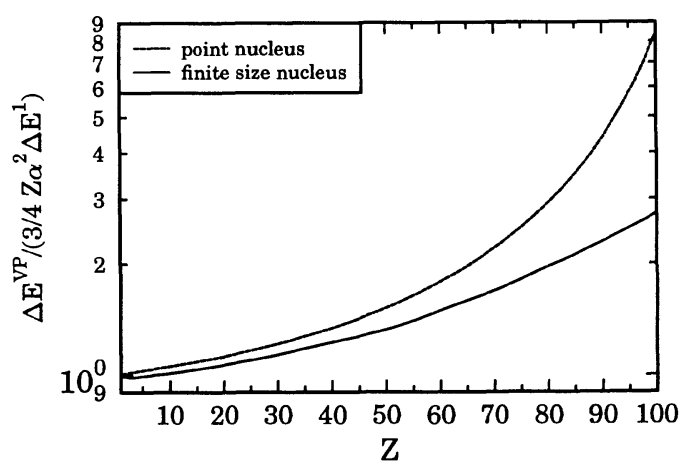

FIG. 5. The total-energy shift $\Delta E^{\mathrm{VP}}$ due to vacuum polarization, normalized to $3 / 4 \alpha(Z \alpha) \Delta E^{1}$, as function of the nuclear charge number $Z$ in the range $1 \leq Z \leq 100$. The dashed line corresponds to the outcome for a point nucleus and the full line signifies the energy shift for an extended nucleus. The evident discrepancy between the exact results and the series expansion prediction can be traced back to the EL term, in which the exact propagator in the Coulomb field is taken into account effectively by employing the wave functions generated by the static potential $V(r)$ supplemented by the Uehling potential. 
up the magnetic and the electric part of the vacuumpolarization part, is plotted for the point-nucleus potential (dashed line) as well as for the potential of a finite-size nucleus (full line). Here, the difference between these two results is most pronounced.

\section{SUMMARY}

We presented a first calculation of the vacuumpolarization contribution to the ground-state hyperfinestructure splitting as a function of the nuclear charge number $Z$. One major uncertainty of our calculation still results from electrostatic couplings within the electronpositron loop.
The computation of the self-energy contribution is in progress. A more ambitious evaluation of the influence of the extended magnetization distribution might be a first essential step in understanding the experimental value of $\Delta \lambda=243.87(4) \mathrm{nm}$ for the ground-state hyperfine splitting of ${ }^{209} \mathrm{Bi}^{+82}$.

\section{ACKNOWLEDGMENTS}

The authors are grateful for interesting and fruitful discussions with T. Kuehl, I. Klaft, and the other members of the experimental group from the Bi-experiment at GSI.
[1] Quantum Electrodynamics, edited by T. Kinoshita (World Scientific, Singapore, 1990), p. $583 \mathrm{ff}$.

[2] A. C. Zemach, Phys. Rev. 104, 1771 (1956).

[3] I. Klaft et al., GSI Report No. 92-1 (unpublished), p. 185.

[4] I. Klaft, S. Borneis, T. Engel, T. Kühl, D. Marx, R. Neumann, S. Schröder, P. Seelig, and L. Völker, GSI Nachrichten No. 08-93 (1993) (unpublished), p. 28.

[5] T. Kühl (private communication).

[6] M. Finkbeiner, B. Fricke, and T. Kühl, Phys. Lett. A 176, 113 (1993); for more details see M. Finkbeiner, Diploma thesis, Kassel, 1992 (unpublished).

[7] S. M. Schneider, J. Schaffner, G. Soff, and W. Greiner, J. Phys. B 26, L581 (1993).

[8] A. Bohr and V. F. Weisskopf, Phys. Rev. 77, 94 (1950).

[9] E. M. Rose, Relativistic Electron Theory (Wiley, New York, 1961).

[10] Y. Ting and D. Williams, Phys. Rev. 89, 595 (1953); P.
Raghavan, At. Data Nucl. Data Tables 42, 189 (1989).

[11] S. M. Schneider, G. Soff, and W. Greiner, J. Phys. B 26, L529 (1993).

[12] H. de Vries, C. W. de Jager, and C. de Vries, At. Data Nucl. Data Tables 36, 495 (1987).

[13] J. Johnson and R. A. Sorensen, Phys. Rev. C 2, 102 (1970).

[14] A. Rüetschli, L. Schellenberg, T. Q. Phan, G. Piller, L. A. Schaller, and H. Schneuwly, Nucl. Phys. A 422, 461 (1984).

[15] N. M. Kroll and F. Pollock, Phys. Rev. 86, 876 (1952).

[16] R. Karplus and A. Klein, Phys. Rev. 85, 972 (1952).

[17] S. J. Brodsky and G. W. Ericson, Phys. Rev. 148, 26 (1966).

[18] J. R. Sapirstein, Phys. Rev. Lett. 51, 985 (1983).

[19] D. E. Zwanziger, Phys. Rev. 121, 1128 (1961).

[20] E. Fermi, Z. Phys. 60, 320 (1930). 